Horizons philosophiques

\title{
La mondialisation comme nouveau visage du monde : paradoxe de l'unité et éclatement
}

\section{Abdenour Berkani}

Volume 15, numéro 2, printemps 2005

Cultures ou mondialisation?

URI : https://id.erudit.org/iderudit/801290ar

DOI : https://doi.org/10.7202/801290ar

Aller au sommaire du numéro

Éditeur(s)

Collège Édouard-Montpetit

ISSN

1181-9227 (imprimé)

1920-2954 (numérique)

Découvrir la revue

\section{Citer cet article}

Berkani, A. (2005). La mondialisation comme nouveau visage du monde : paradoxe de l'unité et éclatement. Horizons philosophiques, 15(2), 15-30. https://doi.org/10.7202/801290ar d'utilisation que vous pouvez consulter en ligne. 


\section{La mondialisation}

\section{comme nouveau visage du monde : paradoxe de l'unité et éclatement}

Depuis une vingtaine d'années nous assistons avec la mondia-lisation à un phénomène complètement nouveau qui bouleverse à tel point notre façon de voir le monde en général et le monde politique en particulier que certains vont jusqu'à parler de

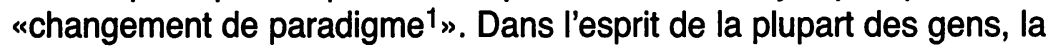
mondialisation demeure l'image abstraite d'un moloch unificateur qui par la voie économique conduirait à uniformiser l'ensemble des modes de vies, des mœurs et des cultures de la planète. J'entends explorer dans ce travail la dimension inverse de la mondialisation qui conduit à une résurgence significative de ce que j'appellerais les «identités particulières". Je voudrais par là montrer toute l'ambiguilté et le caractère duel d'un phénomène dont l'expression se partage en une dialectique entre l'un et le multiple, entre «l'unité et l'éclatement». Si en effet la mondialisation consiste en une tendance de fait, à l'uniformisation des modes de production et de consommation, elle est aussi sur le plan culturel et politique le surgissement d'autres formes et d'autres voies de revendications identitaires aussi riches que variées qui constituent en dernière analyse la mosaïque planétaire. II s'agit alors, au-delà du constat de l'ambivalence de ce phénomène, de se demander en restant à égale distance des alarmistes et des optimistes, ce qui dans ce phénomène est préjudiciable ou au contraire, profitable à la démocratie, tant dans son esprit que dans ses institutions et ses différentes mises en forme. Dans cette optique l'ouvrage de Jean Marie Guéhenno, La fin de la démocratie, semble apporter un éclairage indispensable. Son concept de «libanisation du monde", à la lumière des événements qui dessinent l'espace politique mondial contemporain, prend une pertinence que j'aimerais mettre en exergue afin de mieux comprendre les enjeux d'une pensée politique avertie pour un XXIe siècle qui se cherche. 


\section{La mondialisation ou l'universel en marche}

"Il n'y a rien qui soit d'un bloc dans ce monde, tout y est mosaïque." Malgré ce constat édifiant de Balzac dans la préface à Une fille d'Eve, la mondialisation nous apparaît comme un bloc massif qui écrase la diversité humaine. Dans l'inconscient collectif, en partie nourri par les médias, la mondialisation est représentée comme un moloch uniformisateur qui avale les différences, nivelle la multiplicité du genre humain pour faire régner une universelle unité. Une telle vision engendre un effet claustrophobique. Nous ne sommes plus les habitants d'un monde infiniment riche et varié que toute une vie ne suffirait pas à épuiser, nous avons le sentiment au contraire d'habiter un bocal. Un globe terrestre à taille humaine, pas plus gros qu'un village, et pourrait-on dire pas plus gros qu'une cage. Dans une certaine mesure, une telle vision n'est pas illégitime car la mondialisation se traduit de façon concrète, bien que parfois virtuelle, par les interconnexions des marchés financiers, qui étant régis par les mêmes règles et mécanismes, tendent à former et multiplier des systèmes et des réseaux fermés et uniformes. A. Giddens par exemple définit la mondialisation ainsi : "Ce sont les médias ramifiés à travers le monde, les réseaux et systèmes en général qui obligent à intensifier les relations symboliques et sociales, tout ce qui entraîne l'influence réciproque des événements locaux et des événements lointains ${ }^{2} »$. La mondialisation serait donc une sorte de conspiration immanente de l'ordre social mondial, une conspiration sans conspirateur. Les médias, les entreprises, les divers organismes et institutions, l'ensemble des réseaux qui relient les hommes, seraient animés d'une dynamique interne qui déferlerait telle une avalanche sur l'ensemble des sociétés humaines recouvrant tout du blanc universel de l'immaculée raison capitaliste. La crainte serait donc fondée, et l'action des groupes anti-mondialisation serait légitime. Si par la mondialisation, la modernisation a atteint son stade ultime dans l'effacement des distances, que le proche et le lointain interagissent l'un sur l'autre, alors le règne du même est en route et nous entrons dans l'ère du conformisme global. La question est de savoir quel masque revêt ce conformisme global?

La tentation est grande de confondre mondialisation et américanisation et penser que si conformisme il y a, il est unilatéral : le reste du monde suit l'impulsion américaine, porte ses jeans, écoute sa musique, regarde ses films et boit son Coca-Cola. C'est la vision classique du conformisme. II y a un centre, un élément ou une classe 
dominant qui impose au reste de la société sa façon de voir et de vivre. Le reste des hommes (ou des nations), tel un troupeau de brebis suivrait docilement son berger. Certaines brebis galeuses sortant du rang pour affirmer leur liberté et leur individualité seront dites anticonformistes, rebelles, marginales. Elles susciteront l'admiration secrète de certains, et le mépris affiché de tous. II y a, on ne peut le nier, dans le déploiement de la mondialisation quelque chose de ce conformisme là. Cependant il existe comme le fait remarquer J.M. Guéhenno $^{3}$, un autre type de conformisme qui caractérise notre époque et que l'on pourrait définir non pas comme le suivi de la périphérie à l'égard du centre, mais comme cet art subtil propre à l'homme moderne d'éviter la dissonance, de ramener le différent au semblable et l'autre à soi. C'est selon Guéhenno le conformisme de l'âge relationnel, par opposition à l'âge institutionnel. Si l'âge institutionnel accordait un statut à part à la différence qu'il ordonnait et hiérarchisait, l'âge relationnel dans lequel nous entrons, inclut la différence dans un rapport de relativité. Ce qui était fondamentalement différent devient simplement relatif et s'inscrit dans un rapport de comparaison qui fait ressortir le semblable dans la différence. L'idéal de ce monde là, nous dit Guéhenno : "ce n'est pas le conflit institutionnalisé, mais un calme anticyclonique, que rien ne viendrait perturber, un état stable où l'homogénéité est la règle et la différence l'anomalie ${ }^{4}$ ». II faut alors à tout prix, pour coller à cette injonction insidieuse du conformisme contemporain, éviter la dissonance, calmer les esprits, atténuer les conflits par le dialogue, la concertation, le consensus. Il faut éviter les affrontements ou changements traumatisants pour que les choses de la vie coulent sans douleur les unes dans les autres. Mais ramener l'autre au même, la différence à l'identité, c'est aussi rendre impossible tout anticonformisme. Le conformisme moderne a cette particularité perverse qu'il englobe aussi l'anticonformisme.

L'Universel en marche entraîne donc avec lui tout un cortège de périls. Dans La fin de la démocratie, Guéhenno prophétise non seulement la fin du régime démocratique et la fin de l'État-nation qui en est le seul support moderne connu, mais aussi la fin du politique et la fin du sujet moderne tel que nous les connaissions et définissions jusqu'à présent. Au delà de ce que la thèse peut avoir de séduisant par ce qu'elle a d'inquiétant, il convient tout de même de relativiser ce qui, me semble-t-il, relève d'un mythe, en un sens tout aussi suspect que les mythes millénaristes annonçant la fin du monde pour des 
temps très proches. Car si la mondialisation, en tant que conséquence ou aboutissement de la modernisation est une machine à fabriquer du semblable et fait miroiter le spectre redouté ou espéré de la fin de l'histoire, la réalité des affaires humaines quand à elle rechigne toujours à se plier aux desseins linéaires des théories politiques. On est alors amené à se poser deux questions.

1) Compte tenu des horreurs dont a accouché le siècle de la dissonance des États-nations démocratiques ou non, l'âge du conformisme nécessaire est-il à redouter ou au contraire à appeler de tous nos vœux?

2) Si la mondialisation est l'universel en marche comme nous venons de le voir, n'est-ce pas aussi le mouvement contraire?

Dans I'Homme sans qualités, le romancier Robert Musil, sensible aux soubresauts du monde moderne, nous rappelle superbement les contradictions du réel : «L'époque contemporaine, dit-il, a été dotée d'un très grand nombre d'idées et avec chaque idée, par une attention spéciale du Destin, de l'idée contraire. De sorte que l'individualisme et le collectivisme, le nationalisme et l'internationalisme, le socialisme et le capitalisme, l'impérialisme et le pacifisme, le rationalisme et la superstition, (...). Déjà ce fait nous paraît aussi naturel que l'existence du jour et de la nuit, du chaud et du froid, de l'amour et de la haine et dans le corps humain, de muscles fléchisseurs répondant à leurs contraires les extenseurs ${ }^{5}$ ». Ainsi de même que dans le corps humain les muscles extenseurs ont besoin des muscles fléchisseurs, dans la mondialisation l'Universel ne se peut concevoir sans le particulier.

\section{L'envers du décor : \\ la résurgence des identités particulières}

J'appelle «résurgence des identités particulières, les mouvements de revendication politiques ou juridiques des diverses communautés humaines, petites ou grandes qui face à un ensemble politique dominant luttent pour leur reconnaissance». Ce phénomène, lié à la mondialisation, de résurgence des revendications communautaires s'élevant dans le monde contre les structures rigides des Étatsnations institués, Guéhenno l'appelle «la libanisation du monde». On parle aussi d'ethnicisation, de tribalisation du monde ou encore de balkanisation. J'utilise le terme géographique de résurgence, au sens où ce phénomène des communautés restreintes qui font entendre leur voix à la faveur de la mondialisation, me semble comparable à une 
rivière souterraine qui cheminant silencieusement sous une montagne (ici 200 ans d'État-nation), refait surface de l'autre coté de la montagne sous la forme d'un torrent grondant. II y a donc à mon sens dans la mondialisation un mouvement de balancier entre l'universel et le particulier. Dans un article du journal le monde du 1er juillet 1992, Edgar Morin, constate au sujet de l'Europe, un processus similaire : "ll y a, dit-il, une course de vitesse engagée en Europe entre les processus de dissociation et de désintégration, et ceux d'association et d'intégration". À mesure en effet que l'Europe construit ses édifices postnationaux qui fédèrent les puissances nationales de l'union, d'autres voix subnationales, régionales et locales, longtemps étouffées par la rigidité des structures nationales centralisées, s'élèvent et se font entendre. Certes nous avons hérité des Lumières un paradigme universaliste qui annonçait en même temps que l'avènement des États-nations constitués, la fin des rivalités ethniques, tribales ou communautaires. Marx dira par exemple plus tard que l'ethnicité est un écran de fumée par rapport à la classe. La civilisation moderne, rationnelle et universelle dont la mondialisation est le point d'aboutissement, devait, portée par le mythe d'un progrès croissant des techniques et des savoirs, conduire au dépassement de ces restes d'appartenances obscures et irrationnels. Or force est de constater que si la modernité dans ses premiers élans a eu tendance à illustrer cette idée, la mondialisation en marche semble démontrer au contraire la survivance tenace des identités ethniques. La modernité n'a pas complètement réussi à désenchanter et à rationaliser le monde. Pas plus qu'elle n'a réussi à venir à bout de la religion, elle n'a réussi à arracher les hommes à leur déterminisme socioculturel.

II peut paraître déplacé d'évoquer un concept aussi suranné que celui d'ethnicité alors que l'on réfléchit sur l'avenir. Ethnies, tribus, communautés, ceci relève d'un champ lexical qui semble loin de la réalité politique des États-nations démocratiques actuels. L'âge de la barbarie communautaire ne subsiste à nos yeux que dans certains coins du globe encore primitifs tels que le Moyen Orient ou encore les Balkans. L'affirmation sanglante des identités communautaires telle qu'on l'a vue par exemple au Liban semble étrangère aux démocraties modernes. Que signifie alors le concept étrange de «libanisation du monde" forgé par Guéhenno au chapitre 3 de La fin de la démocratie? Dans les premiers chapitres, Guéhenno fait le constat inquiet mais, dans une certaine mesure, lucide de la fin des nations et de la politique dans l'ère nouvelle de la mondialisation. Le constat est 
alarmant car dans l'horizon des concepts politiques contemporains, «Démocratie, État-nation, et civilisation sont indissociables». Par opposition aux démocraties occidentales, le Liban constitue cet "Orient compliqué», primitif et barbare où en l'absence d'un Etat-nation fort et unifié, à l'image des Etats-nations occidentaux, les différentes communautés se déchirent. Mais sous l'effet de la mondialisation, l'ère des Etats-nations est selon Guéhenno révolue, et le Liban devient le miroir prophétique dans lequel l'occident peut observer sa future donne politique. «Le Liban n'est pas ce pays perdu quelque part sur la carte du Proche-Orient. II est désormais en chacun de nous ${ }^{6}$ ". Or qu'est-ce que le Liban? Un État multinational, multiculturel, multiethnique, multireligieux, aux frontières floues et changeantes où les rapports de force entre les différentes communautés font office de droit.

Mais encore une fois, en quoi cela nous concerne-t-il? Quel rapport entre les États-nations modernes unifiés et démocratiques où règnent l'ordre et la prospérité, et l'anarchie quasi féodale qui règne au Liban? En quoi le Liban constitue-t-il le nouveau paradigme politique des démocraties modernes? Ici encore il convient de citer Guéhenno :

L'archaïsme supposé du Moyen-Orient est donc peut-être plus proche de notre modernité que nous ne voulons bien l'admettre. II montre les dangers de l'âge abstrait, libéré des contraintes de l'espace, dans lequel nous entrons. La puissance ne s'affranchit pas impunément de l'espace et le compromis, qui fut toujours fragile, entre la communauté et la nation est rompu. Là où il n'y a plus nation, il y a communauté, Là où il n'y a plus délimitation d'un territoire, il y a recherche des origines. Si tu ne te définis pas par le lieu où tu vis dis-moi d'où tu viens?

La distinction entre nation et communauté semble fondamentale, et l'illusion des démocraties modernes est de s'être convaincues que les nations civiques qu'elles avaient créées étaient devenues avec la force du temps et de l'éducation, des communautés indivises. Certes l'illusion avait une part de vérité, mais cette part était trop fragile pour résister à l'ère de l'abstraction. Le fond identitaire de la nation n'était pas assez solide pour résister à la dissolution de sa forme spatiale. La tragédie libanaise, (qui, et ce n'est pas un hasard, était il y a pas si longtemps un des pays les plus modernes du Proche-Orient), consiste 
en ceci qu'ayant échoué à former une communauté nationale homogène sur le modèle européen, les différentes communautés sont entrées en guerre les unes contre les autres : «faute d'avoir pu se définir comme une communauté homogène, le Liban a peu à peu commis un suicide collectif». Les luttes d'intérêt et de poúvoir entre les différentes communautés ont conduit à l'éclatement du pays. Fautil conclure de cela que tout le mal vient de la logique communautaire de ces identités meurtrières? Le chaos est-il intrinsèque à la logique communautaire, et l'ordre est-il l'essence exclusive des États-nations?

Pendant plus de cinq siècles, les différentes communautés ethniques et religieuses du Proche-Orient, sous la coupe de l'empire Ottoman, ont cohabité sans trop de problèmes dans cette partie aujourd'hui trouble du monde. Juifs, catholiques et musulmans vivaient sinon dans l'harmonie, pour le moins dans une certaine tolérance mutuelle. Qu'il s'agisse des Grecs ou des Arméniens orthodoxes, ou encore des communautés maronites du Liban ou coptes d'Égypte, chaque communauté se voyait assurée d'une certaine autonomie qui lui permettait non seulement de suivre sa foi mais aussi de s'administrer elle-même. II n'y a avait pas d'État-nation, et les seules frontières étaient celles extérieures de l'empire. Toutes proportions gardées, cela montre que la violence n'est pas inhérente à la logique communautaire. Dès lors, force est de constater que ce qui pose problème, c'est la confrontation de la résurgence des revendications communautaires avec la logique institutionnelle rigide de l'État-nation. C'est avec la chute rapide de l'empire ottoman et l'essor de la colonisation, que l'idée simple de nation une et indivisible, est venue heurter le jeu souple et complexe des communautés enchevêtrées de cette partie du monde. De cette complexité communautaire, le Liban est le miroir le plus révélateur et d'une grande puissance heuristique. Avant la contamination par l'idée européenne de nation, les conflits entre ces différentes communautés étaient de vulgaires escarmouches. Avec la volonté nationale de poser des frontières sous l'équation : une nation = un territoire $=$ une communauté, la complexité relationnelle des communautés du Proche-Orient s'est transformée en un bain de sang. Ne pouvant résoudre cette équation, le Liban a commis «un suicide collectif».

"Les communautés sont devenues à la fois forteresses et prisons; les lignes pointillées qu'on avait voulu tracer entre les États sont apparues à l'intérieur même de l'État, isolant des villages, et distinguant jusqu'aux quartiers d'une même ville. 
Là où l'imbrication était trop grande pour qu'une séparation claire pût être établie, il a fallu par la terreur arriver à la clarté8". C'est ainsi par exemple que pendant la guerre du Chouf, les sunnites isolés au milieu de la communauté chrétienne de la montagne ont été systématiquement assassinés. De même la minorité chrétienne maronite isolée en milieu musulman a été décimée :

Tous ces massacres pour répondre à l'équation nationale, une communauté = une nation = un territoire. On aurait pu ne pas se sentir concernés si ces massacres étaient dû au simple fait de l'existence de ces communautés. Or nous avons vu que ces conflits naissent de l'incompatibilité entre les deux aspirations, communautaires d'une part et nationales d'autre part. Mais si ces deux logiques sont réellement incompatibles, alors précisément parce qu'il y a dans l'occident moderne, résurgence de ces revendications communautaires, aucune démocratie n'est à l'abri d'une libanisation de son territoire. Une erreur encore trop répandue est de croire que les «autres» ce sont «eux», et que "eux" sont extérieurs à "nous". Mais la réalité communautaire des nations les plus unifiées en apparence est plus complexe. Chacun est susceptible à un moment donné dans une société civile d'être désigné comme étant "l'autre", et ce que nous appelons les "autres» ou "eux» sont aussi parmi "nous». II y a en France 4,5 millions de musulmans. La glorieuse république peut-elle encore maintenir, en France mais aussi ailleurs, l'équation magique, une nation = une communauté = un territoire? Et ce par la simple force d'abstraction du statut de "citoyen»? C'est parce que la réponse est des plus incertaines, que le concept de libanisation du monde de Guéhenno me semble être plus qu'une prophétie loufoque lorsqu'il dit que «les massacres du Liban illustrent tragiquement les risques de repli communautaire que porte en elle la crise de l'idée européenne de nation ${ }^{\prime \prime}$.

Enfin si l'on pense encore que le problème est loin, la situation géopolitique des Balkans ces dix dernières années illustre la thèse de Guéhenno, à tel point que l'on a repris la même tournure pour parler de «balkanisation» de l'Europe, phénomène qui n'avait pas échappé en 1995 à Guéhenno lorsqu'il écrit son livre :

La logique communautaire qui a commencé de déchirer l'Europe centrale et orientale n'est pas seulement le réveil de démons historiques un moment gelés par le communisme 
totalitaire. Elle ne s'arrêtera pas nécessairement à nos marges. Sous d'autres formes, elle peut gagner les démocraties les plus avancées, parce qu'elle correspond aussi à l'évolution technologique des économies les plus modernes ${ }^{10}$.

Les événements provoqués dans l'Est de l'Europe par un certain retard de développement dû à un demi siècle de communisme, peuvent être provoqués par la cause inverse en Europe de l'Ouest, à savoir un développement économique et technologique qui affranchit des contraintes de l'espace et déborde les cadres institutionnels établis.

Ainsi comme nous l'annoncions en introduction, la mondialisation semble, comme Janus, offrir un double visage. D'un coté elle rapproche les solitudes et efface les différences mais d'un autre coté elle les ravive et les maintient. L'esprit n'aime pas les paradoxes qui sont la seule pâture féconde de la réalité. Or il n'y a à mon sens paradoxe plus fécond que celui de cette mondialisation qui conjugue l'unité et l'éclatement, qui concilie l'universel et le particulier. L'ennui c'est que nous voulons mettre un ordre à tout cela. II nous faut des formes nettes, bien délimitées, des visions synoptiques sur l'avenir pour faire des projets, fixer des limites et bâtir des institutions pour accoucher d'un nouvel ordre. II nous faut, pour reprendre une expression d'Amin Maalouf, rapprivoiser la panthère».

\section{Apprivoiser la panthère : quel nouvel ordre pour demain?}

«Pour Antonin, nous dit Marc Aurèle, la "cosmopolis" est cet État le plus ancien et le plus digne de tous, au sein duquel les différents États étaient un peu comme des maisons". En effet, la première idée qui nous vient pour donner une forme politique mondiale unifiée au mouvement du monde est l'idée de la République universelle au sens antique et au sens kantien du terme. L'ère confuse dans laquelle nous entrons serait alors le passage d'une société mondiale divisée en plusieurs États, à une société mondiale unifiée sous un seul État politique formant la "cosmopolis», c'est à dire une cité dans laquelle les hommes seraient, quelles que soient leurs origines, tous également "citoyens du monde» et soumis à une même législation. Mais l'idée de République Universelle soulève plusieurs questions qui nous obligent à revenir sur certaines des idées maîtresses de l'héritage kantien avec ses lumières mais aussi ses impasses et ses apories. Dans l'ensemble de ses écrits sur la question, notamment dans «la doctrine du droit»11, Kant nous laisse avec un certain 
nombre d'objections à l'idée du cosmopolitisme, dont il faut rendre compte si l'on veut les dépasser ou pour le moins en sortir. Si le cosmopolitisme n'est ni la SDN, ni l'ONU, sous quelle forme matérialiser l'Idée? Dans quelles institutions incarner le corps sans chair du cosmopolitisme? Quel statut donner à un hypothétique, voire improbable, État cosmopolitique? De quelle réalité sociale sera-t-il le reflet politique? Quels seront son but et son enjeu? S'agira-t-il pour lui simplement de maintenir la paix dans un équilibre tendu des forces ou bien de promouvoir une coopération positive et réelle dans tous les domaines de la vie économique, écologique et culturelle? S'agira-t-il d'une simple "fédération d'États libres» comme le suggère le $2^{\circ}$ article du «Projet de paix perpétuelle» 12 , option qui réitérerait les apories et impasses du droit international, ou au contraire d'une force supranationale, abstraite ou concrète, appuyée sur le droit ou la force, le droit et la force, l'éthique ou la manipulation pour obtenir l'ordre et la paix?

Qu'il s'agisse d'une fédération des nations, dont le siècle passé nous a montré les dérives meurtrières ou bien d'un "grand corps politique futur", dont on entrevoit sans peine les inévitables égarements totalitaires, la question qui se pose lorsque l'on cherche à jeter les fondements d'une République universelle est de savoir, non seulement si c'est possible et souhaitable, mais aussi ce qui restera du politique une fois disparu le décor qui le fait être. On peut fort bien envisager par exemple que le cosmopolitisme s'incarne dans la figure unique d'un seul État qui conserverait la multiplicité des nations avec toute leur diversité. Mais s'il n'y a qu'un seul État, le terme État garderait-il encore sa pertinence? La cosmopolis ne signifierait-elle pas la fin du politique? En essayant de donner une forme politique déjà modélisée et figée dans des institutions préétablies aux contradictions inhérentes aux problèmes politiques mondiaux, ne risquons-nous pas de nous échouer sur la complexité du réel? Pour Guéhenno, cela ne fait aucun doute, chercher à donner une forme institutionnelle à la réalité politique de notre siècle c'est se fourvoyer sur sa nature : «En vérité, il n'y a plus de corps constitués et ceux qui attendent du XXIe siècle l'avènement de la république universelle et la réalisation du rêve de Kant, se trompent profondément ${ }^{13}$ ". Car ce qui se crée n'est pas un grand corps politique mondial, mais «un tissu sans contours apparents, une accrétion indéfinie d'éléments interdépendants ${ }^{14}$ ". Nous entrevoyons avec les objections de Guéhenno à la République universelle, comme un hoquet de l'histoire 
suspendue qui nous ramènerait aux marges d'une organisation politique oubliée par. la modernité, l'empire.

L'empire correspond, dans l'ultime étape de la modernité incarnée dans la mondialisation, au passage de l'âge institutionnel, simple, ordonné, structuré en systèmes à l'âge relationnel, complexe, multiple, multidimensionnel, et déstructuré à l'infini par les réseaux qui le traversent sans lui offrir aucune armature solide. Dès lors à la question : «quelle forme concrète et stable donner à la mondialisation?", nous sommes saisis d'effroi devant un réel qui devient insaisissable. Nous avons l'impression que le passage de l'âge institutionnel à l'âge relationnel annonce le règne du chaos. Mais pour reprendre une phrase de Bergson, le mot désordre ne désigne jamais que la présence d'un ordre qui ne nous intéresse pas ou que l'on ne voit pas. On pourrait alors distinguer entre les deux conceptions traditionnelles de l'ordre : Kosmos et Taxis. L'ordre procédant de la Taxis est un ordre imposant une structure rigoureuse de l'esprit plaquée sur le réel pour lui donner sens. C'est un ordre transcendant, posé d'en haut et qui structure le réel par le rationnel. L'ordre procédant du Kosmos est au contraire un ordre immanent au réel qui nous fait comprendre que le chaos n'est pas si chaotique que cela et qu'il a une forme d'ordre spontané, autoproclamé, qui malgré une apparente anarchie, possède en fait sa propre arché, sa propre structure. II ne faut donc pas confondre une structure interne qui ne nous apparaît pas encore clairement dans l'ordre mondial qui s'annonce, et l'absence d'ordre.

Dès lors, plutôt que de se demander quelle forme on va bien pouvoir lui donner, peut-être serait-il plus juste de se demander quelle forme il tend à vouloir revêtir, compte tenu de sa nature propre. Pour Guéhenno encore une fois, le XXIe siècle sera l'avènement de l'âge impérial qu'il définit comme suit :

Nous appelons l'âge qui vient impérial, d'abord parce qu'il succède à l'État-nation comme l'empire romain a succédé à la république romaine : la société des hommes est devenue trop vaste pour former un corps politique. Les citoyens $y$ forment de moins en moins un ensemble capable d'exprimer une souveraineté collective; ils sont seulement des sujets juridiques, titulaires de droits et soumis à des obligations, dans un espace abstrait aux frontières territoriales de plus en plus indécises...c'est un monde à la fois unifié et privé de centre ${ }^{15}$. 
Cet âge complexe est alors celui d'une puissance diffuse et relationnelle qui rend vaine la recherche d'une forme institutionnelle unifiée qui permette d'ordonner cette complexité. Mais là où il y.a du logos, le chaos fera toujours l'objet d'un projet d'organisation. La raison humaine ne prospère pas dans le désordre, et le monde multidimensionnel, sans structure, sans souveraineté, sans contours nets qui s'offre à ses yeux ne peut que l'effrayer. Et plus les grands problèmes (environnement, drogue, santé publique) prennent une dimension mondiale, plus le besoin devient urgent d'en organiser de manière rigoureuse le traitement. Nous sommes incapables de vivre dans un espace multidimensionnel et mal défini, peut-être est-ce précisément ce talent qu'il nous faudra acquérir ou retrouver pour atteindre la souplesse nécessaire pour vivre dans cette ère nouvelle. Si cette ère nouvelle est plus ouverte, plus souple, plus dynamique et semble-t-il plus libre, qu'est-ce qui fait que l'on a tant de mal à l'accepter et que nous nous agrippons aux vestiges d'une époque révolue? Si la Démocratie fait partie d'un passé moins glorieux que son avenir, pourquoi y tenons nous tant?

Plus haut nous avons posé deux questions. Nous avons répondu à la deuxième qui consistait à nous demander dans quelle mesure la mondialisation n'est pas seulement l'universel en marche mais aussi la résurgence des identités particulières. II s'agit maintenant de tenter de répondre à la première. Compte tenu des horreurs dont a accouché le siècle de la dissonance et le règne de l'âge institutionnel, l'âge du conformisme nécessaire est-il à redouter ou au contraire à appeler de tous nos vœux? Qu'avons-nous à perdre et qu'avons-nous à gagner à un tel changement? La fin de la démocratie est-elle à redouter ou bien à espérer? La question qui nous occupe ici est le rapport de la mondialisation à la démocratie et la chance ou la menace qu'elle constitue pour les valeurs auxquelles nous accordons de l'importance. Schématiquement nous pourrions ramener la Démocratie au triomphe de deux valeurs fondamentales : l'égalité et la liberté, plus le moyen qui permet de les mettre en œuvre à savoir la représentation électorale. Or qu'est-ce qui dans ces trois valeurs est menacé par la mondialisation?. Je me consacrerais essentiellement ici à la question de la liberté. Si la démocratie est vécue comme le triomphe par la représentation de la liberté, elle est aussi ce qui la restreint. II ne s'agit pas ici de confondre la liberté et la licence en souhaitant un retour à une forme de liberté primitive où la liberté serait pour chacun de faire ce qu'il veut. II s'agit juste de remettre en 
situation que la liberté positive acquise dans le cadre de la société civile implique, il ne faut pas l'oublier, une forme de renoncement à une partie de sa liberté déposée entre les mains des institutions publiques. Si l'on considère qu'une liberté sans sécurité est une illusion, incontestablement, cette liberté positive est un grand bien. Mais dans l'âge relationnel, cette liberté ne s'évanouit pas, elle change simplement de garant. À partir du moment où l'organisation en réseaux de l'âge relationnel l'emporte sur la structure pyramidale de l'âge institutionnel, la liberté change de main et semble plus garantie par l'interface des relations entretenues avec autrui que par une institution établie.

À l'âge des réseaux, la relation privilégiée que le citoyen entretenait avec les institutions démocratiques chargées de le représenter et de défendre ses intérêts devient secondaire. Car l'essentiel des réseaux de connexions et de relations qu'il entretient dans sa vie se passe en dehors d'elles. C'est ainsi que la légitimité du politique comme principe organisateur de la vie des hommes est ébranlée. Non seulement la politique est secondaire, mais elle constitue même parfois un frein ou un obstacle au bon vivre ensemble des citoyens qu'elle entend lier. La politique jadis réalisant la liberté des hommes par leur socialisation pour éviter qu'ils ne s'entre-tuent, devient aujourd'hui une chaîne et un fardeau. La question est alors de savoir si l'on doit lui rester fidèle, par loyauté, reconnaissants, pour ce que par le passé elle nous a apporté, ou bien lui tenir rigueur d'être aujourd'hui un frein à une liberté peut-être plus grande? Si par le passé, la démocratie était le garant de l'intérêt général, est-elle aujourd'hui indispensable? Cet intérêt ne se définit-il pas de lui même dans le jeu subtil des interactions humaines? En définitive a-t-on encore besoin de la démocratie? Pour répondre à cette question, on peut distinguer deux niveaux de définition de la liberté démocratique.

1) Tout d'abord la liberté démocratique c'est le droit pour une collectivité de prendre en main son destin et de se doter d'une organisation politique autonome, d'un gouvernement qui puisse exprimer sa volonté collective.

2) Ensuite la liberté en démocratie, c'est le droit qu'a chaque homme de se protéger des abus du pouvoir, liberté d'où découle par exemple pour les minorités nationales, la garantie de ne pas être opprimées par la majorité.

II y aurait donc une liberté qui concerne le groupe et une qui concerne l'individu. Avec l'âge impérial, la première conception 
meurt. D'une certaine manière la liberté est aussi une invention de l'âge institutionnel liée à l'idée d'un centre, ne serait-ce que celui du sujet. Aura-t-elle encore un sens dans l'âge relationnel sans centre ni sujet? Si la liberté implique un sujet, la disparition du sujet implique la disparition de la liberté. On arrive à l'ère de ce que Jean Padioleau appelle la tyrannie des petites décisions. Décisions qui en fragmentant le pouvoir font disparaître en même temps le sentiment de forte coercition qui caractérise l'âge institutionnel. Dès lors que le pouvoir s'émiette, son poids se délite et il n'apparaît plus comme la source de la domination d'un sujet qui de toute manière n'existe plus en tant qu'objet sur lequel un pouvoir centralisé peut avoir une prise coercitive. L'ère de la fin de la Démocratie serait-elle alors porteuse de plus de liberté? En un sens oui. Puisque avec la disparition d'un pouvoir central fort, disparaît toute forme de coercition clairement identifiable. Mais c'est une liberté, qui comme toute chose dans l'âge relationnel, n'est que relative. Guéhenno nous dit à ce propos : "La grande surprise de cet âge est que la plupart d'entre nous s'y sentent bien. Nous avons le sentiment de n'avoir jamais été aussi libres. Et nous plaignons nos ancêtres soumis à toutes sortes de contraintes qui ne nous touchent plus. Qu'il s'agisse des films que nous regardons, des vêtements que nous portons, et ce qui est plus important, des sentiments que nous éprouvons, il n'y a plus guère d'interdiction. Pourquoi cette liberté laisse-t-elle un goût de cendres dans la bouche? ${ }^{16}$ ".

Ce goût de cendres, c'est le revers de bâton de l'âge relationnel. Si notre liberté n'est plus muselée par l'institution, elle est en revanche épiée par les milliers de regards des autres qui constituent ce nouveau pouvoir qui irrémédiablement détermine une bonne partie de notre comportement et donc de l'essentiel de ce qu'il reste de notre moi. À quoi sommes-nous enchaînés? aux regards des autres, et ces regards sont à la fois, juges, victimes, bourreaux et barreaux d'une prison qui pour être abstraite n'en est pas moins réelle.

En conclusion comme en introduction nous pouvons dire que le $21^{e}$ siècle qui s'ouvre, est le siècle des contradictions et des paradoxes de toutes sortes. Si libre, si ouvert et pourtant si enchaîné. Notre pouvoir sur lui ne consistera pas à dépasser ses tensions mais au contraire à les maintenir. Notre seule prise résidera dans l'art subtil que nous posséderons (ou pas) de transformer ses contradictions ruineuses en horizons féconds. Car dans cette tension permanente entre l'universel et le particulier incarné par la mondialisation et que 
nous avons essayé de restituer ici, c'est l'avenir de notre condition humaine, donc politique qui se joue. Les marges incertaines de ce siècle dessinent une humanité à la fois nouvelle et semblable à ce qu'elle a toujours été. Citons pour finir un passage du songe de Nabuchodonosor, roi de Sumer, qui me semble illustrer parfaitement l'ambiguïté de ce siècle qui vient sur nous plus que nous n'allons vers lui : «Le quatrième royaume, dit-il sera fait moitié de fer, moitié d'argile. Divisé, il sera moitié fort et moitié fragile. Les pieds, partie fer et partie argile de potier : le royaume sera partie fort et partie fragile. Mais assurément ce royaume ne tiendra pas, aussi sûr que le fer et l'argile ne peuvent se mêler ensemble». Sans doute la tâche nous incombe-t-elle d'inventer les matériaux nouveaux qui feront tenir ensemble les contradictions du monde.

\author{
Abdenour Berkani \\ Doctorant, \\ Université de Montréal et \\ Université de Lyon 3 (Jean-Moulin)
}




\section{Abdenour Berkani}

1. A. Hurrel, "Kant and the Kantian Paradigm in International Relations", Review of Intemational Studies, 16, 1998, p. 183-205.

2. A. Giddens, The Consequences of Modemity, Cambridge, University Press, 1990, p. 64 traduction libre.

3. Jean-Marie Guéhenno, La fin de la démocratie, chap. 6, «le conformisme nécessaire», Paris, Flammarion, 1992.

4. Ibid., p. 107.

5. Robert Musil, L'homme sans qualités, Paris, Seuil, 1995, p. 469.

6. Jean-Marie Guéhenno, op. cit., p. 59.

7. Sur le système ottoman des millets, voir le commentaire de Kymlicka au chapitre 8 de La citoyenneté multiculturelle, Montréal, Éd. Boréal,2001.

8. Jean-Marie Guéhenno, op. cit., p. 63.

9. Ibid., p. 64.

10. Ibid., p. 67.

11. Emmanuel Kant, La doctrine du droit, trad. A. Philonenko, Paris, Librairie philosophique Vrin, 1993.

12. Emmanuel Kant, Projet de paix perpétuelle, in CEuvres philosophiques, Paris, Gallimard, collection bibliothèque de la pléiade, 1986.

13. Jean-Marie Guéhenno, op. cit., p. 74.

14. Ibid., p. 86.

15. Ibid., p. 13.

16. Ibid., p. 104. 